\title{
CONTINUED MONITORING OF LANDSAT REFLECTIVE BAND CALIBRATION USING PSEUDO-INVARIANT CALIBRATION SITES
}

\author{
Julia A. Barsi, Brian L. Markham, Dennis L. Helder
}

\author{
Science Systems and Applications, Inc., NASA/GSFC Code 618, Greenbelt MD 20771; NASA/GSFC \\ Code 618, Greenbelt MD 20771, South Dakota State University, Brookings SD 57007
}

\begin{abstract}
Though both of the current Landsat instruments, Landsat-7 Enhanced Thematic Mapper+ (ETM+) and Landsat-5 Thematic Mapper (TM), include on-board calibration systems, since 2001, pseudo-invariant calibration sites (PICS) have been added to the suite of metrics to assess the instruments' calibration. These sites do not provide absolute calibration data since there are no ground measurements of the sites, but in monitoring these PICS over time, the relative calibration can be tracked. The sites used by the Landsat instruments are primarily in the Saharan Desert.
\end{abstract}

To date, the trending from the PICS sites has confirmed that most of the degradation seen in the ETM+ on-board calibration systems is likely not degradation of the instrument, but rather degradation of the calibration systems themselves. However, the PICS data show statistically significant degradation (at 2-sigma) in all the reflective spectral bands of up to $-0.22 \%$ /year since July 2003 . For the TM, the PICS were instrumental in updating the calibration in 2007 and now suggest two bands may require another update. The data show a statistically significant degradation (at 2-sigma) in Bands 1 and 3 of -0.27 and $-0.15 \% /$ year, respectively, since March 1999. The data filtering and processing methods are currently being reviewed but these PICS results may lead to an update in the reflective band calibration of both Landsat-7 and Landsat-5.

Index Terms - Landsat, reflective bands, radiometric calibration, pseudo-invariant calibration sites, ETM+, TM

\section{INTRODUCTION}

The two operational Landsat instruments, the Enhanced Thematic Mapper+ $(\mathrm{ETM}+)$ and the Thematic Mapper (TM) both have on-board calibration (OBC) systems. The TM OBC consists of an internal lamps and a shutter, which provides a per-scan estimate of gain and bias [2]. The $\mathrm{ETM}+$ has a similar lamp system, as well as a full aperture solar diffuser panel, which is used monthly [4]. While the OBC methods provide very precise gain estimates (on the order of tenths of a percent), they have limitations due to their own instability. When changes are observed in the instrument's response to an $\mathrm{OBC}$, it is often difficult to determine whether the change is due to the calibrator or from the imager. Vicarious calibrations assist in verifying the on-board calibration but are much lower precision and are generally labor intensive. Teams from the University of Arizona and South Dakota State University have been collecting data to validate the calibration of both TM and ETM+ since 1999, though they only average about six collects per year for each team [7].

Since 2001, the Landsat calibration has also been monitored using Pseudo-Invariant Calibration Sites (PICS) recommended by Cosnefroy [1]. Though not a source of absolute calibration, the PICS provide a long-term fullaperture stable source. This effort has led to an update of the Landsat-5 TM reflective band calibration in 2007 [2] and increased confidence that the changes seen in the response of Landsat-7 ETM+ to its on-board calibration system are due to the calibrators and not the imager [5].

\section{LANDSAT PSEUDO-INVARIANT CALIBRATION SITES}

Previous studies evaluated largely vegetation-free desert regions, including portions of the Saharan and Arabian deserts for use as calibration sites for optical sensors [1]. The list of sites established by Cosnefroy et al served as a starting point in 2001 for the Landsat effort to incorporate Pseudo-Invariant Calibration Sites (PICS) into the monitoring system. Landsat data covering these sites were ordered and assessed for uniformity, cloud-cover and availability in the archive. From the 20 initial Cosnefroy sites, four were chosen for long-term analysis. In the Landsat WRS-2 Path/Row coordinate system, they are $181 / 40,177 / 45,165 / 47$ and 201/46 which are located in Libya, Epypt/Sudan, Saudi Arabia, and Mauritania respectively.

In subsequent years, the PICS analysis was extended to include Landsat-5 TM. The 181/40 site was the only one of the four sites for which there was significant coverage in the global archive so the Landsat-5 analysis was initially based 
on data from a single site. An additional site, 192/39, was added to the analysis after several years, because the $181 / 40$ imagery was generally saturated in the TM band 5 (SWIR1).

A $90 \times 90 \mathrm{~km}$ region of interest in the center of the each scene was established to eliminate some non-uniform features at the edges of some sites. This same size region was used for all sites regardless of scene content. For ETM+, the region was centered on the nominal scene center for the individual Path/Row. This means that as the satellite moves east/west across the path, the same region of the PICS is used every time. However, the geometric knowledge for TM was less precise when this analysis was started, so the region of interest is based on the pixel center of the image, not on a specific geographic location.

For ETM+, the data were processed using standard processing techniques to calculate radiance. For the TM analysis, the $90 \times 90 \mathrm{~km}$ regions were extracted as biassubtracted digital counts and the calibration gains and biases are applied outside of the standard processing software to convert to radiance ${ }^{1}$. The top-of-atmosphere radiances were converted to top-of-atmosphere reflectances [3]. An empirical correction for solar elevation angle is applied to the reflectances (Figure 1), to attempt to account for some of the systematic seasonal variations most likely due to a scene bidirectional reflectance distribution function (BRDF) effect. Studies are ongoing to generate corrections for changing atmospheric conditions and a physical model of the BRDF. The average reflectances of the region of interest are scaled to gain for comparison to measurements made by the on-board calibrators. For ETM+, the data are scaled to the prelaunch gains and for TM, the gains are scaled to the gain from ETM+/TM cross-calibration [6].

\section{RESULTS}

Figures 2 and 3 illustrate the utility of the PICS sites in the calibration of both ETM+ and TM. Use of the PICS has allowed confirmation that the decreasing gains estimated by the ETM+ solar panel is likely due to changes in the panel and not due to a change in the detectors (Figure 2). Table 1 compares the estimated change in calibration gain since July 2003 as determined by the solar panel and PICS. The PICS estimate is calculated from the weighted average of the gain change over time estimated by each of the four sites (192/39 is not included). The estimate of gain change is only for data acquired since July 2003 as that is when the degradation of

\footnotetext{
${ }^{1}$ The standard processing system was originally not used for TM processing because the calibration gains were not trusted and the calibration gain could not be adjusted within the standard software. Since the 2007 update of the calibration gains, the processing of the TM PICS data could be run through standard processing but the switch has not been made yet.
}

the solar panel changed characteristics, and the thought was perhaps the PICS and panel data would agree. However, while the ETM+ response to the solar panel is changing by as much as $0.7 \%$ /year, the ETM+ response to the PICS only changing by as much as $-0.22 \% /$ year. The change is statistically significant in all bands at a 2-sigma level. Currently, a review of the data filters and corrections is underway, but these results could lead to an update in the calibration gains for the whole history of the ETM+.

For Landsat-5, the PICS data were used to revise the whole calibration history of the TM reflective bands. After one calibration update based on internal calibrator data in 2001, the PICS study revealed that the trend predicted by the lamps was due to both instrument and lamp degradation. An update was made to the calibration gains in 2007, based almost entirely on the PICS data to remove the effect of the lamp degradation from the calibration [2]. Since the 2007 update, the PICS continue to indicate that the TM reflective bands are relatively stable (Figure 3 ), while the lamp system has become so erratic as to be unusable in most bands. Table 1 lists the gain change over time as predicted by the 181/40 site when processed using the 2007 calibration gains for data acquired after March 1999. March 1999 was chosen as the starting point because that is when the degradation that started just after launch appeared to level off. However, the trends show that the $-0.27 \%$ and $-0.15 \% /$ year change in Bands 1 and 3 is statistically significant and hence the 2007 Calibration Gains may need to be updated to reflect the continuing decline. However, the data populations are not evenly distributed over the time period since March 1999; from 1999 through 2001, the US archive only holds summer data of $181 / 40$, so the whole annual distribution of sun angles is not available yet. An attempt will be made to fill in the calibration record before making any changes to the calibration.

Table 1. Landsat-7 ETM+ and Landsat-5 TM gain change over time as estimated by the PICS and the ETM+ solar panel. The ETM+ results are only calculated on data since July $2003(\sim 4.5$ year since launch), when the scan line corrector failed. The TM results are for data acquired since March 1999 ( 15 years since launch) for 181/40 only; the trend for the second site (192/39) has not been fully populated yet.

\begin{tabular}{|c|c|c|c|}
\hline & $\begin{array}{c}\text { Landsat-7 } \\
\text { ETM+ } \\
\text { Solar Panel } \\
\text { [\%/year } \pm \\
\text { 2-sigma] }\end{array}$ & $\begin{array}{c}\text { Landsat-7 } \\
\text { ETM+ } \\
\text { PICS } \\
{[\% / \text { year } \pm} \\
\text { 2-sigma] }\end{array}$ & $\begin{array}{c}\text { Landsat-5 TM } \\
\text { PICS (181/40 } \\
\text { only) } \\
\text { [\%/year } \pm \\
\text { 2-sigma] }\end{array}$ \\
\hline Band 1 & $-0.51 \pm 0.02$ & $-0.22 \pm 0.06$ & $-0.27 \pm 0.05$ \\
\hline Band 2 & $-0.46 \pm 0.02$ & $-0.10 \pm 0.05$ & $0.03 \pm 0.06$ \\
\hline Band 3 & $-0.52 \pm 0.04$ & $-0.10 \pm 0.05$ & $-0.15 \pm 0.06$ \\
\hline Band 4 & $-0.68 \pm 0.04$ & $-0.11 \pm 0.09$ & $-0.03 \pm 0.12$ \\
\hline Band 5 & $-0.11 \pm 0.01$ & $-0.17 \pm 0.05$ & $\mathrm{~N} / \mathrm{A}$ \\
\hline Band 7 & $0.13 \pm 0.02$ & $-0.20 \pm 0.10$ & $0.02 \pm 0.18$ \\
\hline Band 8 & $-0.56 \pm 0.02$ & $-0.09 \pm 0.08$ & \\
\hline
\end{tabular}




\section{CONCLUSIONS}

Though not an absolute calibration system, the PICS continue to be used to monitor the calibration of Landsat-7 ETM+ and Landsat-5 TM in a relative sense and to corroborate or refute changes seen in the on-board calibration systems. The PICS have already been used to update the calibration of the TM in 2007.

The latest analysis indicates degradation in both ETM+ and TM. In ETM+, all bands are showing statistically significant degradation (at 2-sigma) of between - 0.10 and $0.22 \% / y e a r$. The data filtering and corrections will be reviewed thoroughly before making any changes, but a change to the lifetime calibration of ETM+ is possible.

In the TM, two bands are showing statistically significant degradation (at 2-sigma) of -0.27 and $-0.15 \%$ /year, but the distribution of the data over time may be contributing to the significance. An attempt will be made to fill out the history completely before making any changes.

\section{REFERENCES}

[1] H. Cosnefroy, M. Leroy, and X. Briottet, "Selection and characterization of Saharan and Arabian desert sites for the calibration of optical satellite sensors," Remote Sensing of Environment, Vol. 58, pp. 101-114, 1996.

[2] D.L. Helder, B.L. Markham, K.J. Thome, J.A. Barsi, G. Chander, and R. Malla, "Updated Radiometric Calibration for the Landsat-5 Thematic Mapper Reflective Bands," IEEE Transactions on Geoscience and Remote Sensing, Vol. 46, No. 10, pp. 3309-3325, Oct 2008.

[3] Landsat 7 Science Data Users Handbook, Section 11.3.2 http://landsathandbook.gsfc.nasa.gov/data_prod/prog_sect11_3.html,

Accessed Jun 1, 2011.

[4] B.L. Markham, J.L. Barker, E. Kaita, J. Seiferth, and R. Morfitt, "On-Orbit performance of the Landsat-7 ETM+ radiometric calibrators," International Journal of Remote Sensing, Vol 24, No 2, 2003.

[5] B.L. Markham, K.J. Thome, J.A. Barsi, E. Kaita, D.L. Helder, J.L. Barker, P.L. Scaramuzza, "Landsat-7 ETM+ on-orbit reflective-band radiometric stability and absolute calibration," IEEE Transactions on Geoscience and Remote Sensing, Vol 42, No 12, pp. 2810-2820, Dec 2004.

[6] P.M Teillet, J.L Barker, B.L Markham, R.R Irish, G Fedosejevs, J.C Storey, Radiometric cross-calibration of the Landsat-7 ETM+ and Landsat-5 TM sensors based on tandem data sets, Remote Sensing of Environment, Volume 78, Issues 1-2, October 2001, Pages 39-54, ISSN 0034-4257, 10.1016/S00344257(01)00248-6.

[7] K.J. Thome, D.L. Helder, D. Aaron, J.D. Dewald, "Landsat-5 TM and Landsat-7 ETM+ Absolute Radiometric Calibration Using the Reflectance-Based Method," IEEE Transactions on Geoscience and Remote Sensing, Vol 42, No 12, pp. 2777-2785, Dec 2004.

Figure 1. The empirical correction to reflectance for solar elevation angle. The figure on the left shows the gain vs. solar elevation angle for the original data and the corrected data. The figure on the right is the ratio of the corrected to the original. The data are adjusted to a reference solar elevation angle of 52.5 for ETM+ and 58.5 for TM using a linear regression of gain vs. angle (different angles were used because originally, the TM data did not cover an entire annual cycle). The correction is as large as 3.5\% for some sun angles in ETM+ Band 7. The correction removes some of the annual cyclical pattern, but not all the systematic patterns are removed. Studies to characterize and correct for the remaining non-random variation are ongoing: BRDF and atmospheric effects are being investigated.
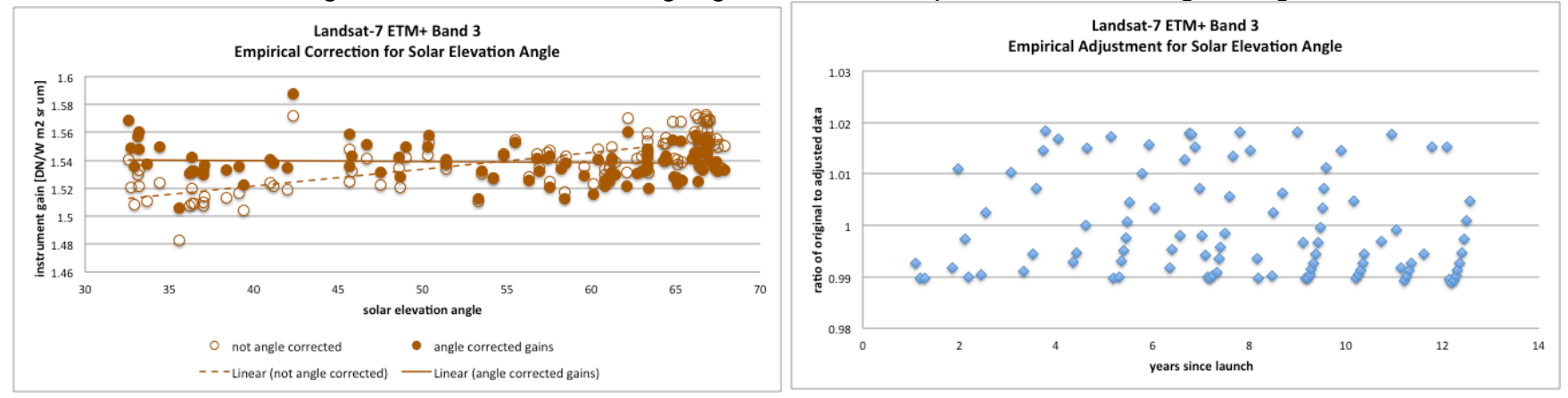
Figure 2. The Landsat-7 ETM+ Band 3 calibration gains estimated by the PICS and the full-aperture solar calibrator over the life of the mission. The changing response to the panel is attributed to ultra-violet exposure rather than a change in the instrument since the response to the PICS has been more stable. The weighted average of the gain change of each of the four sites estimates that this band is degrading by $-0.10 \% /$ year.

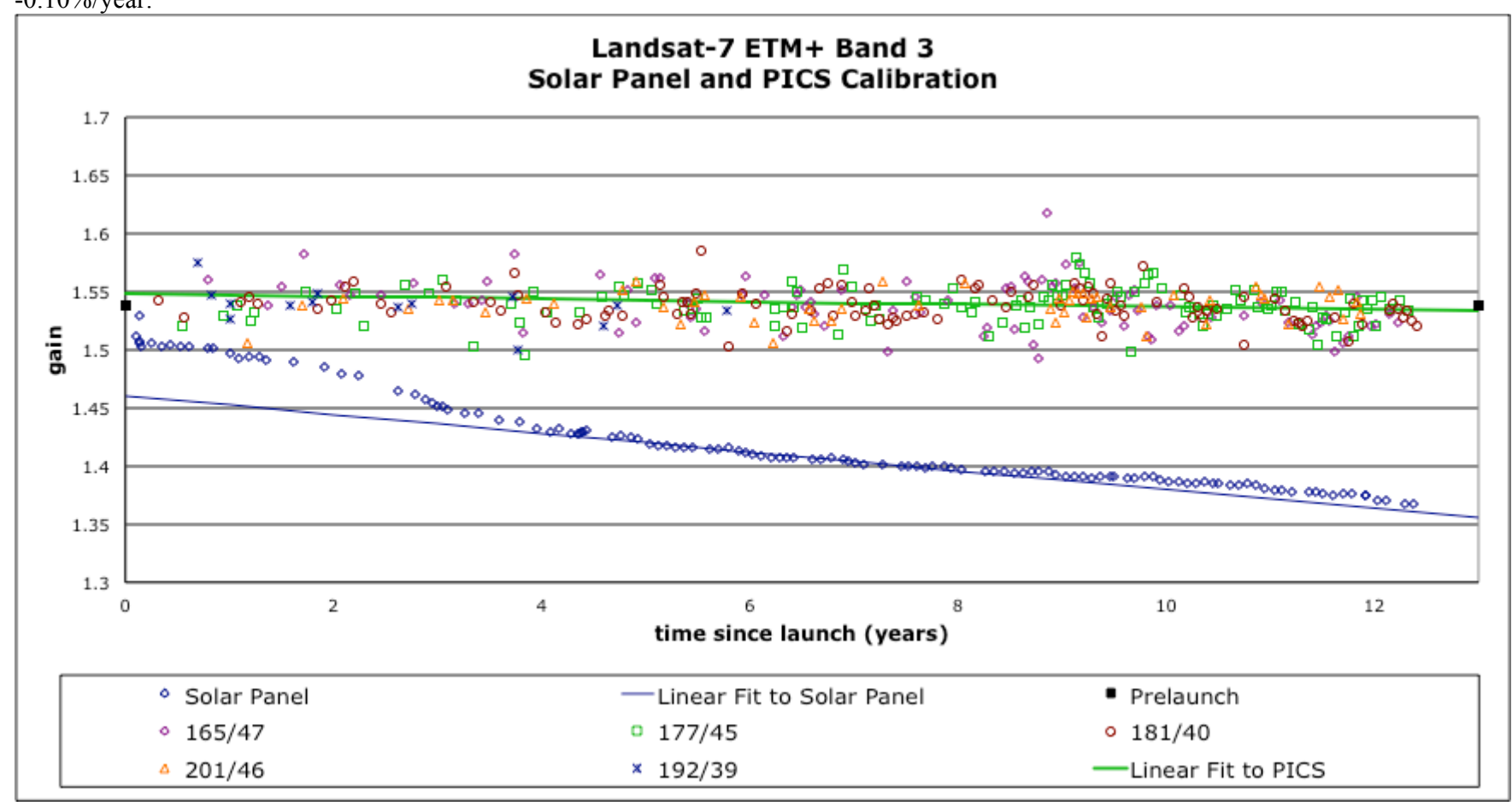

Figure 3. The Landsat-5 TM Band 3 calibration gains as estimated by the PICS for the lifetime of the mission. The internal calibration onboard TM is too unstable to use for calibration now, so the PICS are the primary source for relative long term calibration. Two sites are included on the figure, though not enough data has been assessed for 192/39 to use it as a source yet. The PICS data estimate that this band is degrading by $-0.15 \%$ year.

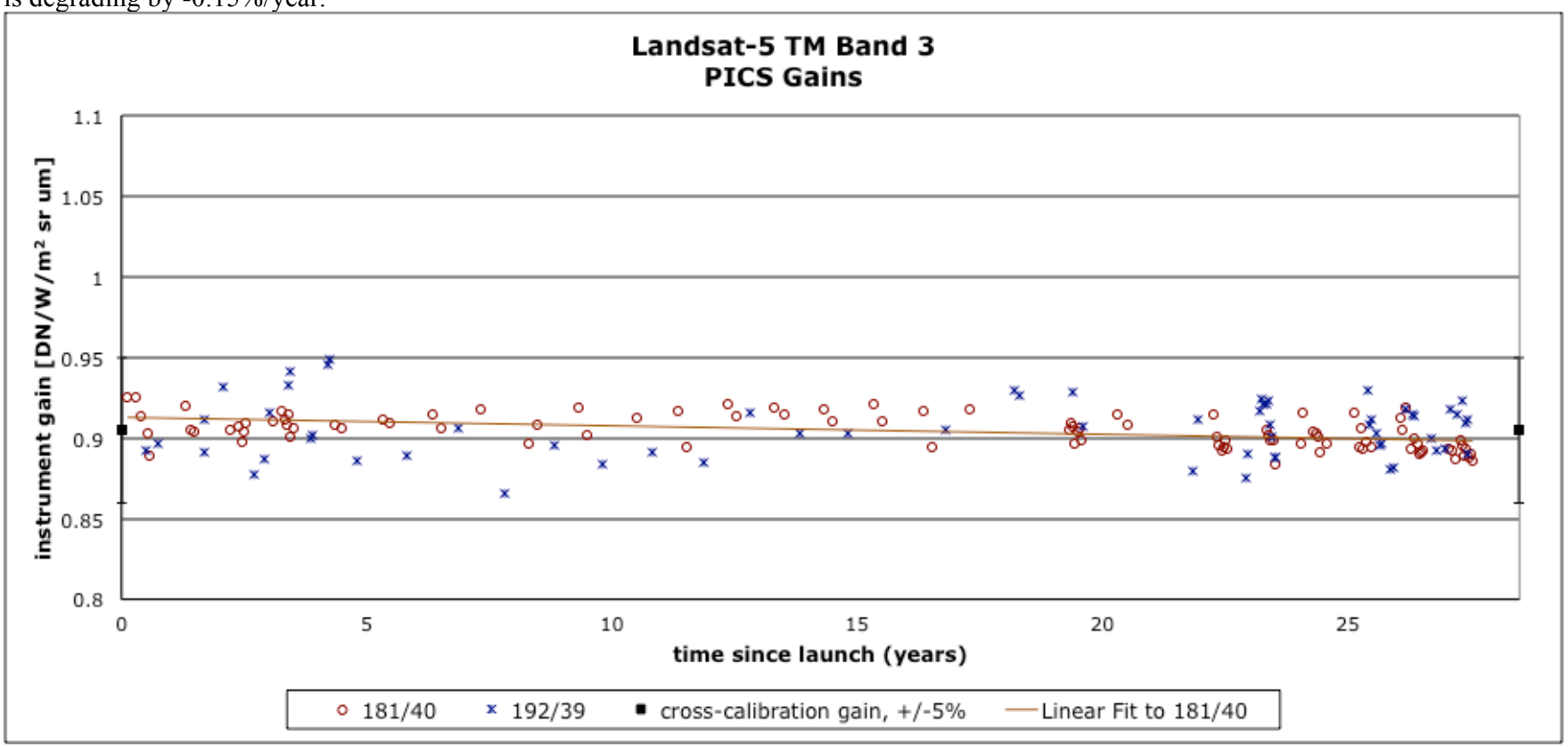

